\title{
Evaluación de Dos Niveles de Energía Digestible en base a los Estándares Nutricionales del NRC (1995) en Dietas de Crecimiento para Cuyes (Cavia porcellus L)
}

\author{
Evaluation of Two Levels of Digestible Energy based on Nutritional Standards \\ of the NRC (1995) in Growth Diets for Guinea Pigs (Cavia porcellus L)
}

\author{
Felix Esteban Airahuacho Bautista ${ }^{1}$, Víctor Vergara Rubín ${ }^{2}$
}

\section{Resumen}

\begin{abstract}
El objetivo de la investigación fue evaluar los niveles de 2.7 y 2.9 Mcal de energía digestible (ED)/kg de alimento y densidades de aminoácidos (DA) de 100, 110 y $120 \%$ en relación a los estándares nutricionales del NRC (1995) sobre el rendimiento productivo de cuyes de genotipo mejorado durante la fase de crecimiento posdestete (2-6 semanas de edad). Las dietas se formularon considerando la concentración de aminoácidos en relación al nivel de ED (g/Mcal) con inclusión de vitamina $\mathrm{C}$ en el alimento peletizado, debido a que no se utilizó forraje verde. Se utilizó un diseño completamente al azar para todos los tratamientos y un arreglo factorial de $2 \times 3$, teniendo como factores dos niveles de ED $(2.7$ y $2.9 \mathrm{Mcal} / \mathrm{kg})$ y tres DA $(100,110$ y $120 \%)$, sin considerar el tratamiento control. Se realizó el análisis de covariancia, considerando al peso inicial como covariable, y el ajuste de Bonferroni para determinar diferencia entre tratamientos y factores. Los tratamientos más eficientes para conversión alimenticia fueron $3.0 \mathrm{Mcal} \mathrm{ED} / \mathrm{kg}$ (control) y $2.9 \mathrm{Mcal} \mathrm{ED} / \mathrm{kg}, 120 \%$ DA (3.38 y 3.32, respectivamente), mientras el tratamiento 2.7 Mcal ED $/ \mathrm{kg}, 100 \%$ DA fue el menos eficiente (3.82). En el arreglo factorial, el factor 2.9 $\mathrm{Mcal} \mathrm{ED/kg}$ mejoró en ganancia de peso, consumo de alimento, conversión alimenticia y rendimiento de carcasa, y sin diferencias por efecto del factor DA. El peso de las vísceras (hígado, riñón y corazón) no fue diferente entre tratamientos.
\end{abstract}

Palabras clave: energía digestible, NRC, cuyes

\footnotetext{
${ }^{1}$ Escuela de Ingeniería Zootécnica, Universidad Nacional José Faustino Sánchez Carrión, Huacho, Lima, Perú

${ }^{2}$ Departamento Académico de Nutrición, Universidad Nacional Agraria La Molina, Lima, Perú

${ }^{3}$ E-mail: Estebanfelix1107@hotmail.com
}

Recibido: 2 de julio de 2016

Aceptado para publicación: 23 de febrero de 2017 
The aim of this research was to assess levels of 2.7 and 2.9 Mcal of digestible energy (ED/kg of feed) and amino acids densities (DA) of 100, 110 and $120 \%$ in relation to nutritional standards of the NRC (1995) on productive performance of genotype improved guinea pigs after weaning (2-6 weeks of age). Diets were formulated considering the amino acids concentration relative to the level of ED (g/Mcal) with inclusion of vitamin C in the pelleted feed because no fresh forage was used. A randomized complete design for all treatments and a factorial arrangement $2 \times 3$ were used, having as factors two levels of ED (2.7 and 2.9 Mcal $/ \mathrm{kg}$ ) and three DA (100, 110 and 120\%), and without considering the control treatment. Covariance analysis was performed, considering the initial weight as a covariate, and Bonferroni adjustment to determine the difference between treatments and factors. The more efficient treatments for feed conversion were 3.0 Mcal ED/kg (control) and 2.9 Mcal ED/kg, 120\% DA (3.38 and 3.32, respectively), while treatment 2.7 Mcal ED/kg, 100\% DA was the less efficient (3.82). Data analysis in the factorial arrangement showed that the $2.9 \mathrm{Mcal} \mathrm{ED} / \mathrm{kg}$ factor improved weight gain, feed intake, feed conversion and carcass yield and without differences by the effect of the DA factor. The weight of viscera (liver, kidney, and heart) showed no statistical difference.

Key words: digestible energy, NRC, guinea pig

\section{INTRODUCCIÓN}

En el Perú se han obtenido genotipos de cuy (Cavia porcellus) de elevados rendimientos productivos como resultado del mejoramiento genético al que ha sido sometido; sin embargo, estos logros no han sido acompañados con suficientes estudios relacionados con sus necesidades de nutrientes y programas de alimentación, lo que ha conllevado a formular raciones utilizando requerimientos establecidos por la National Research Council (1995). Estas formulaciones tienen el inconveniente que los requerimientos han sido determinados en animales criados en condiciones de laboratorio, con un genotipo y medio ambiente muy diferentes y una tasa de crecimiento inferior a los cuyes de crianza comercial en el país.

La satisfacción de las necesidades energéticas con un adecuado nivel de nutrientes, especialmente aminoácidos, es una de las metas de una crianza eficiente. Por otro lado, y debido a los pequeños márgenes de ganancia con que se trabaja, se hace necesaria la búsqueda de niveles nutricionales mínimos que promuevan la máxima producción. Según el NRC (1978), los requerimientos de energía para cuyes son de $3.0 \mathrm{Mcal} \mathrm{ED} / \mathrm{kg}$; no obstante, resultados de investigación en el Perú señalan que $2.90,2.86$ y $2.86 \mathrm{Mcal} \mathrm{ED} / \mathrm{kg}$ serían adecuados para las etapas de crecimiento-engorde, gestación y lactación, respectivamente (Caicedo, 1985; citado por Correa, 1988).

En términos de proteína y aminoácidos, los esfuerzos están enfocados en determinar el requerimiento adecuado de lisina y metionina más cistina o aminoácidos azufrados (AA). El NRC (1995) determina el requerimiento mínimo de proteína, lisina y AA en $18.0,0.84$ y $0.60 \%$, respectivamente. En el Perú, evaluando dos niveles de ED (2.9 y 3.0 Mcal) y proteína (15 y 18\%), se obtuvo los mejores rendimientos con los animales que consumieron dietas con $18 \%$ de proteína (Torres, 2006); mientras que en un estudio con varios niveles de lisina y AA en dietas isoenergéticas se encontraron los mejores rendimientos al utilizar niveles entre 0.78 a $0.84 \%$ de lisina y de 0.71 a $0.79 \%$ de AA (Remigio, 2006). 
Cuadro 1. Cantidad calculada de proteína y aminoácido por Mcal de energía digestible (g/Mcal) para cada tratamiento

\begin{tabular}{lccccccc}
\hline & \multicolumn{7}{c}{ Tratamientos $^{1}$} \\
\cline { 2 - 8 } & Control & I & II & III & IV & V & VI \\
\hline $\begin{array}{l}\text { Energía digestible, } \\
\text { Mcal/kg }\end{array}$ & 3.0 & 2.7 & 2.7 & 2.7 & 2.9 & 2.9 & 2.9 \\
$\begin{array}{l}\text { Densidad de } \\
\text { aminoácidos, \% NRC }\end{array}$ & 100 & 100 & 110 & 120 & 100 & 110 & 120 \\
Proteína (g/Mcal) & 60 & 60 & 66 & 72 & 60 & 66 & 72 \\
Lisina (g/Mcal) & 2.80 & 2.80 & 3.10 & 3.40 & 2.80 & 3.10 & 3.40 \\
Met + Cist (g/Mcal) & 2.00 & 2.00 & 2.20 & 2.40 & 2.00 & 2.20 & 2.40 \\
Metionina (g/Mcal) & 1.20 & 1.20 & 1.32 & 1.44 & 1.20 & 1.32 & 1.44 \\
Arginina (g/Mcal) & 4.00 & 4.00 & 4.40 & 4.80 & 4.00 & 4.40 & 4.80 \\
Treonina (g/Mcal) & 2.00 & 2.00 & 2.20 & 2.40 & 2.00 & 2.20 & 2.40 \\
Triptofano (g/Mcal) & 0.60 & 0.60 & 0.66 & 0.72 & 0.60 & 0.66 & 0.72 \\
\hline
\end{tabular}

${ }^{1}$ Dos niveles de energía digestible y tres densidades de aminoácidos frente a un grupo control

La densidad de nutrientes, término referido a la cantidad de nutrientes suministrados en relación con la cantidad de energía suministrada (Troesch et al., 2015), se vislumbra como una metodología adecuada para evaluar nuevos patrones de crecimiento. Por otro lado, considerando que el nivel energético del alimento afecta sustancialmente los resultados del comportamiento productivo, sumado a mayores incrementos de peso y rendimiento de carcasa con el uso de líneas genéticas mejoradas de cuyes, se hace necesario evaluar densidades de nutrientes mayores a los recomendados por el NRC (1995). Por ello, el objetivo de la presente investigación fue evaluar el efecto de los niveles de 2.7 y 2.9 Mcal ED por kg de alimento y densidad de aminoácidos (DA) de 100,110 y $120 \%$ en relación a los estándares nutricionales del NRC (1995) en dietas comerciales para cuyes de líneas mejoradas en fase de crecimiento sobre su comportamiento productivo.

\section{Materiales y Métodos}

La investigación fue realizada entre agosto y setiembre de 2005 en las instalaciones de la granja de cuyes de Cieneguilla del Programa de Investigación y Proyección Social en Carnes de la Universidad Nacional Agraria de La Molina (UNALM). Se utilizaron 84 animales machos, destetados de $14 \pm$ 2 días, distribuidos en siete tratamientos de tres replicaciones cada una. La unidad experimental estuvo formada por cuatro animales. El periodo experimental tuvo una duración de siete semanas, del destete hasta las nueve semanas de edad. Se evaluaron dos niveles de ED $(2.7$ y $2.9 \mathrm{Mcal} / \mathrm{kg})$ y tres DA $(100,110$ y $120 \%)$ en relación a los estándares nutricionales del NRC (1995) de $3.0 \mathrm{Mcal} / \mathrm{kg}$, que fue considerado como tratamiento control (Cuadro 1), donde el $100 \%$ de DA se refiere a los gramos de aminoácidos por Mcal de ED recomendado por el NRC (1995) en tres niveles de $\operatorname{ED}(3.0,2.7$ y $2.9 \mathrm{Mcal} / \mathrm{kg})$, 
Cuadro 2. Composición porcentual y nutricional de las dietas experimentales en base fresca

\begin{tabular}{|c|c|c|c|c|c|c|c|}
\hline & \multicolumn{7}{|c|}{ Tratamientos $^{1}$} \\
\hline & Control & I & II & III & IV & V & VI \\
\hline $\begin{array}{l}\text { Energía } \\
\text { digestible, } \\
\text { Mcal/kg }\end{array}$ & 3.0 & 2.7 & 2.7 & 2.7 & 2.9 & 2.9 & 2.9 \\
\hline $\begin{array}{l}\text { Densidad de } \\
\text { aminoácidos, \% } \\
\text { (NRC, 1995) }\end{array}$ & 100 & 100 & 110 & 120 & 100 & 110 & 120 \\
\hline \multicolumn{8}{|l|}{ Componentes } \\
\hline $\begin{array}{l}\text { Subproducto de } \\
\text { trigo }\end{array}$ & 4.97 & 83.64 & 83.51 & 82.24 & 19.96 & 9.48 & 9.40 \\
\hline Torta de soya 47 & 17.56 & 7.45 & 10.82 & 14.49 & 16.00 & 20.35 & 23.18 \\
\hline Maíz & 44.78 & 5.86 & 2.44 & 0.00 & 42.52 & 41.64 & 38.83 \\
\hline Pasta de algodón & 5.61 & 0.00 & 0.00 & 0.00 & 5.17 & 8.22 & 7.66 \\
\hline Harina de alfalfa & 22.70 & 0.00 & 0.00 & 0.00 & 12.00 & 16.00 & 16.50 \\
\hline $\begin{array}{l}\text { Carbonato de } \\
\text { calcio }\end{array}$ & 0.98 & 1.59 & 1.58 & 1.55 & 1.32 & 1.19 & 1.21 \\
\hline Aceite vegetal & 2.50 & 0.58 & 0.78 & 0.87 & 2.13 & 2.23 & 2.30 \\
\hline Sal & 0.43 & 0.40 & 0.40 & 0.40 & 0.44 & 0.44 & 0.44 \\
\hline $\begin{array}{l}\text { Premezcla vit. y } \\
\text { minerales }\end{array}$ & 0.37 & 0.38 & 0.37 & 0.35 & 0.36 & 0.35 & 0.38 \\
\hline Vitamina $\mathrm{C}$ & 0.10 & 0.10 & 0.10 & 0.10 & 0.10 & 0.10 & 0.10 \\
\hline \multicolumn{8}{|c|}{ Composición nutricional $^{2}$} \\
\hline Materia seca, \% & 87.95 & 87.65 & 87.39 & 87.60 & 88.02 & 87.18 & 87.89 \\
\hline Proteína & 19.23 & 16.63 & 16.60 & 17.86 & 17.56 & 19.45 & 20.25 \\
\hline Fibra cruda & 8.46 & 9.48 & 9.50 & 9.24 & 8.75 & 8.64 & 8.69 \\
\hline Extracto etéreo & 5.45 & 4.08 & 3.71 & 3.75 & 4.68 & 5.04 & 5.00 \\
\hline Cenizas & 5.64 & 5.97 & 5.74 & 5.85 & 5.53 & 5.47 & 5.71 \\
\hline Extracto libre $\mathrm{N}$ & 49.17 & 51.49 & 51.84 & 50.90 & 51.50 & 48.58 & 48.24 \\
\hline
\end{tabular}

${ }^{1}$ Dos niveles de energía digestible y tres densidades de aminoácidos frente a un grupo control

${ }^{2}$ Análisis químico proximal realizado en el Laboratorio de Evaluación Nutricional de Alimentos - UNALM

mientras el 110 y $120 \%$ de DA es el incremento de estos aminoácidos recomendados en 10 y $20 \%$.

Las dietas, formuladas según las recomendaciones del NRC (1995) y considerando la DA (concentración de aminoácidos en relación al nivel de ED [g/Mcal]), fueron de- sarrolladas al mínimo costo y los alimentos fueron peletizados en la Planta de Alimentos de la UNALM (Cuadro 2). Se utilizó vitamina $\mathrm{C}$ comercial con un aporte de $35 \mathrm{mg}$ por $100 \mathrm{~g}$ de alimento, para prevenir la enfermedad carencial por la no utilización de forraje verde. El alimento, en la forma física de pellets ( $4.5 \mathrm{~mm}$ de diámetro por $10 \mathrm{~mm}$ de longitud) 
Cuadro 3. Comportamiento productivo ${ }^{1}$ de cuyes alimentados con siete dietas durante siete semanas a partir de los 14 días de edad

\begin{tabular}{|c|c|c|c|c|c|c|}
\hline \multicolumn{2}{|c|}{ Tratamientos ${ }^{\mathrm{A}}$} & \multirow{2}{*}{$\begin{array}{c}\text { Covariable } \\
\text { Peso } \\
\text { inicial }(\mathrm{g})\end{array}$} & \multirow{2}{*}{$\begin{array}{l}\text { Peso final } \\
\text { (g) }\end{array}$} & \multirow{2}{*}{$\begin{array}{c}\text { Ganancia } \\
\text { de peso } \\
(\mathrm{g})\end{array}$} & \multirow{2}{*}{$\begin{array}{c}\text { Consumo } \\
\text { MS } \\
\text { (g) }\end{array}$} & \multirow{2}{*}{$\begin{array}{l}\text { Conversión } \\
\text { alimenticia }\end{array}$} \\
\hline $\begin{array}{c}\text { ED } \\
(\mathrm{Mcal} / \mathrm{kg})\end{array}$ & $\begin{array}{c}\text { DA } \\
(\%, \text { NRC })\end{array}$ & & & & & \\
\hline 3.0 & 100 & 357 & $1149^{a}$ & $16.2^{\mathrm{a}}$ & $2673^{a}$ & $3.38^{\mathrm{a}}$ \\
\hline 2.7 & 100 & 353 & $1023^{a}$ & $13.7^{\mathrm{a}}$ & $2552^{\mathrm{a}}$ & $3.82^{b}$ \\
\hline 2.7 & 110 & 354 & $1076^{\mathrm{a}}$ & $14.7^{\mathrm{a}}$ & $2695^{\mathrm{a}}$ & $3.74^{\mathrm{ab}}$ \\
\hline 2.7 & 120 & 355 & $1072^{\mathrm{a}}$ & $14.4^{\mathrm{a}}$ & $2654^{\mathrm{a}}$ & $3.71^{\mathrm{ab}}$ \\
\hline 2.9 & 100 & 354 & $1106^{\mathrm{a}}$ & $15.3^{\mathrm{a}}$ & $2606^{\mathrm{a}}$ & $3.47^{\mathrm{ab}}$ \\
\hline 2.9 & 110 & 355 & $1106^{\mathrm{a}}$ & $15.3^{\mathrm{a}}$ & $2580^{a}$ & $3.44^{\mathrm{ab}}$ \\
\hline 2.9 & 120 & 360 & $1122^{\mathrm{a}}$ & $15.5^{\mathrm{a}}$ & $2523^{a}$ & $3.32^{\mathrm{a}}$ \\
\hline \multirow{2}{*}{\multicolumn{2}{|c|}{$\begin{array}{l}\text { Factor Energía } \\
\text { digestible }^{\mathrm{B}}\end{array}$}} & 2.7 & $1059^{b}$ & $14.3^{b}$ & $2635^{b}$ & $3.75^{b}$ \\
\hline & & 2.9 & $1110^{a}$ & $15.4^{\mathrm{a}}$ & $2568^{a}$ & $3.41^{\mathrm{a}}$ \\
\hline \multirow{3}{*}{\multicolumn{2}{|c|}{$\begin{array}{l}\text { Factor Densidad de } \\
\text { aminoácidos }^{B}\end{array}$}} & 100 & $1067^{\mathrm{a}}$ & $14.5^{\mathrm{a}}$ & $2581^{a}$ & $3.64^{\mathrm{a}}$ \\
\hline & & 110 & $1092^{a}$ & $15.0^{\mathrm{a}}$ & $2638^{a}$ & $3.59^{\mathrm{a}}$ \\
\hline & & 120 & $1094^{\mathrm{a}}$ & $14.9^{\mathrm{a}}$ & $2586^{\mathrm{a}}$ & $3.52^{\mathrm{a}}$ \\
\hline \multicolumn{7}{|c|}{ Probabilidad } \\
\hline \multicolumn{2}{|c|}{$\begin{array}{l}\text { Energía digestible } \\
\text { (ED) }\end{array}$} & & 0.031 & 0.022 & 0.440 & 0.000 \\
\hline \multicolumn{2}{|c|}{$\begin{array}{l}\text { Densidad de } \\
\text { aminoácidos (DA) }\end{array}$} & & 0.497 & 0.559 & 0.240 & 0.315 \\
\hline \multicolumn{2}{|l|}{$E D \times D A$} & & 0.545 & 0.585 & 0.040 & 0.848 \\
\hline
\end{tabular}

${ }^{A}$ Diseño completamente al azar - ancova ${ }^{B}$ Factorial $2 \times 3$ - ancova

${ }^{a b}$ Letras diferentes dentro de columnas y variables indican diferencia significativa $(p<0.05)$

${ }^{1}$ Valores promedios de tres replicaciones por tratamiento, cuatro cuyes por replicación

y el agua fresca y limpia fue suministrado a voluntad. El análisis químico proximal de las dietas fue realizado en Laboratorio de Evaluación Nutricional de Alimentos (LENA) de la UNALM, utilizando el método AOAC (1990), como criterio de determinación de la humedad, proteína, grasa, fibra y cenizas (Cuadro 2).

Los parámetros evaluados fueron la ganancia de peso (diferencia entre el peso inicial y el peso vivo al final de cada periodo), el consumo de alimento (diferencia entre lo ofrecido y el residuo de cada semana) y la con- versión alimenticia (consumo de alimento dividido entre la ganancia de peso). Además, se determinó el rendimiento de carcasa (incluye piel, patas y órganos nobles) y el peso de órganos nobles (corazón, hígado y riñón), utilizando un cuy de cada unidad experimental. El beneficio de los cuyes se hizo después de 24 horas de ayuno. Todos los pesos fueron registrados utilizando una balanza de mesa digital de $2 \mathrm{~kg}$ de capacidad y con $2 \mathrm{~g}$ de sensibilidad. El diseño estadístico empleado fue el completamente al azar (DCA), con siete tratamientos (dietas experimentales) y tres repeticiones. Además, un DCA con arreglo 
Cuadro 4. Ingesta de ED y nutrientes en cuyes en fase de crecimiento (g/Mcal de ED) y que fueran alimentados con siete dietas durante siete semanas

\begin{tabular}{|c|c|c|c|c|c|c|c|}
\hline & \multicolumn{7}{|c|}{ Tratamientos $^{1}$} \\
\hline & Control & I & II & III & IV & V & VI \\
\hline $\mathrm{ED}, \mathrm{Mcal} / \mathrm{kg}$ & 3.0 & 2.7 & 2.7 & 2.7 & 2.9 & 2.9 & 2.9 \\
\hline DA, \% NRC & 100 & 100 & 110 & 120 & 100 & 110 & 120 \\
\hline ED, Mcal & 8.02 & 6.89 & 7.28 & 7.17 & 7.56 & 7.48 & 7.32 \\
\hline Proteína, $\mathrm{g} / \mathrm{Mcal}^{3}$ & 72.8 & 70.2 & 70.3 & 75.5 & 68.8 & 77.0 & 79.4 \\
\hline Lisina, $\mathrm{g} / \mathrm{Mcal}^{2}$ & 3.2 & 3.2 & 3.6 & 3.9 & 3.2 & 3.6 & 3.8 \\
\hline Met + cist., g/Mcal ${ }^{2}$ & 2.2 & 2.3 & 2.5 & 2.8 & 2.3 & 2.5 & 2.7 \\
\hline Fibra, g/Mcal ${ }^{3}$ & 32.0 & 40.1 & 40.2 & 39.1 & 34.3 & 34.2 & 34.0 \\
\hline $\begin{array}{l}\text { Extracto etéreo, } \\
\mathrm{g} / \mathrm{Mcal}^{3}\end{array}$ & 20.7 & 17.3 & 15.7 & 15.9 & 18.4 & 19.9 & 19.7 \\
\hline
\end{tabular}

${ }^{1}$ Dos niveles de energía digestible y tres densidades de aminoácidos frente a un grupo

${ }^{2}$ Valores tomados del contenido nutricional teórico (Mixit -2)

${ }^{3}$ Valores tomados del análisis químico proximal

factorial 2 x 3 (dos niveles de ED $x$ tres niveles de DA), sin considerar el tratamiento control. Se realizó el análisis de covariancia, considerando al peso inicial como covariable, $\mathrm{y}$ el ajuste de Bonferroni para determinar la diferencia entre los tratamientos y los factores (Field, 2009).

\section{Resultados y Discusión}

No hubo diferencias estadísticas entre tratamientos para el peso vivo final y la ganancia de peso diario, pero en el análisis factorial se encontraron diferencias por efecto del factor $\operatorname{ED}(\mathrm{p}<0.05)$; siendo superior el peso vivo y la ganancia de peso de los cuyes alimentados con dietas de $2.9 \mathrm{Mcal} \mathrm{ED} / \mathrm{kg}$ (Cuadro 3). Los menores incrementos de peso con dietas de $2.7 \mathrm{Mcal} \mathrm{ED} / \mathrm{kg}$ se deberían a la menor cantidad de ED ingerida, posiblemente deficiente para los procesos de síntesis cárnica. Morales et al. (2011), utilizando cuyes de la raza Perú, no encontró diferencias para la ganancia de peso en animales alimentados con dietas isonutritivas de $2.8 \mathrm{y}$ 3.0 Mcal ED/kg.

Analizando el consumo de nutrientes, se observa que los cuyes que consumieron dietas de $2.7 \mathrm{Mcal} \mathrm{ED} / \mathrm{kg}$ muestran una ingestión de energía promedio inferior en $0.4 \mathrm{Mcal}$ y $1 \mathrm{Mcal}$, comparado con la dieta control y $2.9 \mathrm{Mcal} \mathrm{ED} / \mathrm{kg}$, respectivamente; resultados que se relacionarían con la mayor ingestión de fibra (Cuadro 4). Con relación al factor DA, solo se observa tendencias a mayores pesos al incrementar en $20 \%$ más la DA en dietas con $2.9 \mathrm{Mcal} \mathrm{ED} / \mathrm{kg}$, no ocurriendo lo mismo con dietas de $2.7 \mathrm{Mcal} \mathrm{ED} / \mathrm{kg}$ (Cuadro 3). Con una alimentación de solo balanceado con diferentes niveles de lisina y AA en dietas isoenergéticas $(2.75 \mathrm{Mcal} / \mathrm{kg}) \mathrm{se}$ reportaron ganancias diarias de $14.1 \mathrm{~g}$ (Remigio, 2006), similar a los resultados de la presente investigación $(2.70 \mathrm{Mcal} / \mathrm{kg}=14.3 \mathrm{~g})$, mientras que bajo una alimentación mixta se reportan ganancias de 13.19 g/día $(0.89 \%$ lisina, $0.64 \%$ AA y $3.0 \mathrm{Mcal} \mathrm{ED} / \mathrm{kg}$ ) y $12.70 \mathrm{~g} /$ día (0.93\% lisina, $0.66 \%$ AA. y 2.9 Mcal ED/kg) (Cerna, 1997; Torres, 2006). 
Cuadro 5. Efecto de la energía digestible y densidad de nutrientes ${ }^{1}$ sobre el rendimiento de carcasa y peso de corazón, hígado y riñones en cuyes alimentados con siete dietas durante siete semanas a partir de los 14 días de edad

\begin{tabular}{|c|c|c|c|c|c|c|}
\hline \multicolumn{2}{|c|}{ Tratamientos ${ }^{\mathrm{A}}$} & \multirow{2}{*}{$\begin{array}{l}\text { Peso final } \\
\text { (g) }\end{array}$} & \multirow{2}{*}{$\begin{array}{c}\text { Rendimiento } \\
\text { carcasa } \\
(\%)\end{array}$} & \multirow{2}{*}{$\begin{array}{l}\text { Corazón } \\
\text { (g) }\end{array}$} & \multirow{2}{*}{$\begin{array}{l}\text { Hígado } \\
\text { (g) }\end{array}$} & \multirow{2}{*}{$\begin{array}{l}\text { Riñones } \\
\text { (g) }\end{array}$} \\
\hline $\begin{array}{c}\text { ED } \\
\mathrm{Mcal} / \mathrm{kg}\end{array}$ & $\begin{array}{c}\text { DA } \\
(\%, \mathrm{NRC})\end{array}$ & & & & & \\
\hline 3.0 & 100 & $1161^{a}$ & $71^{\mathrm{a}}$ & $6.0^{\mathrm{a}}$ & $42.7^{\mathrm{a}}$ & $12.0^{\mathrm{a}}$ \\
\hline 2.7 & 100 & $925^{\mathrm{a}}$ & $69^{a}$ & $4.0^{\mathrm{a}}$ & $41.3^{\mathrm{a}}$ & $11.3^{\mathrm{a}}$ \\
\hline 2.7 & 110 & $1065^{\mathrm{a}}$ & $67^{\mathrm{a}}$ & $5.3^{\mathrm{a}}$ & $44.0^{\mathrm{a}}$ & $12.7^{\mathrm{a}}$ \\
\hline 2.7 & 120 & $1109^{a}$ & $68^{\mathrm{a}}$ & $6.0^{\mathrm{a}}$ & $50.7^{\mathrm{a}}$ & $14.0^{\mathrm{a}}$ \\
\hline 2.9 & 100 & $1081^{\mathrm{a}}$ & $69^{a}$ & $6.0^{\mathrm{a}}$ & $46.7^{\mathrm{a}}$ & $12.0^{\mathrm{a}}$ \\
\hline 2.9 & 110 & $1207^{\mathrm{a}}$ & $70^{\mathrm{a}}$ & $6.7^{\mathrm{a}}$ & $48.0^{\mathrm{a}}$ & $16.7^{\mathrm{a}}$ \\
\hline 2.9 & 120 & $1007^{\mathrm{a}}$ & $71^{\mathrm{a}}$ & $4.7^{\mathrm{a}}$ & $38.7^{\mathrm{a}}$ & $12.0^{\mathrm{a}}$ \\
\hline \multirow{2}{*}{ Factor $\mathrm{ED}^{\mathrm{B}}$} & 2.7 & $1033^{b}$ & $68^{b}$ & 5.0 & 45.2 & 12.7 \\
\hline & 2.9 & $1099^{a}$ & $70^{\mathrm{a}}$ & 6.0 & 44.8 & 13.6 \\
\hline \multirow{3}{*}{ Factor DA ${ }^{\mathrm{B}}$} & 100 & $1110^{a}$ & $67^{\mathrm{a}}$ & $4.9^{\mathrm{a}}$ & $43.8^{a}$ & $11.7^{\mathrm{a}}$ \\
\hline & 110 & $1062^{\mathrm{a}}$ & $69^{a}$ & $5.9^{\mathrm{a}}$ & $45.9^{\mathrm{a}}$ & $14.7^{\mathrm{a}}$ \\
\hline & 120 & $1025^{\mathrm{a}}$ & $69^{a}$ & $5.5^{\mathrm{a}}$ & $45.0^{\mathrm{a}}$ & $13.0^{\mathrm{a}}$ \\
\hline \multicolumn{7}{|l|}{ Probabilidad } \\
\hline \multicolumn{2}{|c|}{ Energía digestible (ED) } & 0.278 & 0.050 & 0.170 & 0.892 & 0.555 \\
\hline \multicolumn{2}{|c|}{ Densidad nutrientes (DA) } & 0.504 & 0.784 & 0.392 & 0.900 & 0.279 \\
\hline \multicolumn{2}{|l|}{$\mathrm{ED} \times \mathrm{DA}$} & 0.466 & 0.153 & 0.176 & 0.174 & 0.287 \\
\hline
\end{tabular}

A Diseño completamente al azar - ancova ${ }^{B}$ Factorial $2 \times 3$ - ancova

${ }^{a b}$ Letras diferentes dentro de columnas y variables indican diferencia significativa $(p<0.05)$

${ }^{1}$ Valores promedios de tres replicaciones por tratamiento, un cuy por replicación

El consumo de alimento en base a materia seca (CMS) no mostró diferencias entre tratamientos; sin embargo, se encontraron diferencias por efecto del factor ED $(\mathrm{p}<0.05)$, observándose mayores consumos con dietas de 2.7 Mcal ED/kg (Cuadro 3). Estos mayores consumos estarían relacionados con el mayor contenido de fibra en estas dietas, toda vez que este nutriente influye indirectamente sobre el consumo de alimento y la utilización de muchos nutrientes (Mateos et al., 2012). Torres (2006) reporta diferencias de CMS en cuyes que consumieron dietas de 2.8 y $3.0 \mathrm{Mcal} \mathrm{ED} / \mathrm{kg}$ y $18 \%$ de $\mathrm{PB}$, bajo sistema de alimentación mixta; sin em- bargo, Morales et al. (2011) utilizando cuyes de la raza Perú, no encontraron diferencias de CMS en animales alimentados con dietas isonutritivas de 2.8 y $3.0 \mathrm{Mcal} \mathrm{ED} / \mathrm{kg}$.

Al mantener el 100\% de DA recomendado por el NRC (1995) y con diferentes niveles de ED, los cuyes consumieron la misma cantidad de lisina y AA por Mcal de ED (3.2 y $2.3 \mathrm{~g}$, respectivamente), pero hubo un menor consumo con la dieta de $2.7 \mathrm{Mcal}$ ED/ $\mathrm{kg}$ (DA: $100 \%$ NRC), posiblemente debido a la mayor ingesta de fibra bruta en este tratamiento, que actuaría como un limitante físico (Cuadro 4). También se observa una tenden- 
cia a menor consumo cuando se incrementa la DA en 10 y $20 \%$ en dietas de $2.9 \mathrm{Mcal}$ $\mathrm{ED} / \mathrm{kg}$, lo que sugiere la satisfacción de aminoácidos, debido a que la característica nutricional del alimento determina el consumo (McDonald, 1995). El consumo de alimento muestra una interacción ED x DA, debido a las diferencias encontradas por efecto de la ED; sin embargo, esto no fue observado para los efectos de la DA, donde los niveles de 100, 110 y $120 \%$ tuvieron un comportamiento similar.

La conversión alimenticia acumulada más eficiente fue observada en cuyes alimentados con el tratamiento control y $2.9 \mathrm{ED} / \mathrm{kg}$ $-120 \%$ DA $(\mathrm{p}<0.01)$, siendo el menos eficiente el mostrado por el tratamiento $2.7 \mathrm{ED} / \mathrm{kg}$ $100 \%$ DA, cuyo perfil de aminoácidos está por debajo de lo recomendado por el NRC (1995). Además, por efecto del factor ED, los tratamientos con $2.7 \mathrm{Mcal} / \mathrm{kg}$, que poseen mayores contenidos de fibra bruta, mostraron las conversiones menos eficientes con relación a las dietas con $2.9 \mathrm{Mcal} / \mathrm{kg}$ (Cuadro 3). Esto se debería a que los cuyes alimentados con un menor nivel de energía incrementaron el consumo de alimento para cubrir su requerimiento energético, pero este incremento no se vio reflejado en un mayor incremento de peso, alcanzando una pobre conversión alimenticia. Alvarez et al. (2007), evaluando el efecto del nivel y tipo de fibra sobre el rendimiento productivo en conejos en crecimiento reportan deterioro de la conversión alimenticia en $8.8 \%$ en grupos alimentados con el mayor nivel de fibra (18.6\% FDA). En cerdos, sin embargo, los grupos alimentados con dietas de mayor nivel de fibra mostraron menores ganancias de peso, sin afectar la conversión alimenticia (Kallabis y Kaufmann et al., 2012).

La DA no tuvo efecto sobre la conversión alimenticia, pero a mayor densidad de nutrientes (10 y $20 \%$ más con relación al NRC), se observaron tendencias a mejores conversiones alimenticias. Evaluando niveles de lisina y AA en cuyes, Remigio (2006), obtuvo conversiones alimenticias de 3.6 con ni- veles de lisina de 0.78 y $0.84 \%$ y AA de 0.71 y $0.79 \%$, manteniendo una relación de AA/ Lis. entre 91 a $94 \%$. En la presente investigación, la conversión alimenticia promedio fue de 3.4 con dietas que mantuvieron una relación $\mathrm{AA} / \mathrm{Lis}$. de $70 \%$. En aves, la lisina es uno de los aminoácidos que ejerce el efecto más específico en la composición de carcasa y crecimiento muscular (Tesseraud et al., 1996), y su alto contenido en la dieta maximiza la ganancia de peso corporal y mejora la conversión alimenticia (Leclerq, 1998), mientras que altos niveles de metionina en aves de 21 a 42 días de edad influenciaron positivamente la ganancia de peso, consumo de alimento y la conversión alimenticia (Schutte y Pack, 1995; Pesti et al., 1999; Albino et al., 1999).

No se encontró diferencias para el rendimiento de carcasa entre tratamientos, pero el factor ED fue significativo, encontrándose mayores rendimientos de carcasa en los cuyes que consumieron dietas con $2.9 \mathrm{Mcal} \mathrm{ED} / \mathrm{kg}$ $(\mathrm{p}=0.05)$ (Cuadro 5). Morales et al. (2011) alimentando cuyes raza Perú, de 8 semanas de edad, con dietas 2.8 y $3.0 \mathrm{Mcal} \mathrm{ED} / \mathrm{kg}$ mostraron rendimientos de carcasa estadísticamente similares que variaron entre 71 y $72.8 \%$. En cerdos, sin embargo, Beaulieu et al. (2009) reportaron un mayor rendimiento de carcasa en grupos alimentados con dietas de $3.57 \mathrm{Mcal} \mathrm{ED} / \mathrm{kg}$ comparado con la de $3.09 \mathrm{Mcal} \mathrm{ED} / \mathrm{kg}$.

La obtención de dietas balanceadas con 2.7 Mcal ED $/ \mathrm{kg}$ y $9.5 \%$ de fibra cruda implicó la utilización de ingredientes de naturaleza voluminosa ( $83 \%$ de subproducto de trigo), que se reflejó en un mayor consumo de alimento, posiblemente debido al aporte deficiente de ED de la dieta. Esto estuvo relacionado a una mayor ingestión de fibra cruda (Cuadro 4), un posible mayor desarrollo del tracto gastrointestinal y, por tanto, menores rendimientos de carcasa. Un aumento en la ingesta de fibra aumenta el peso del tracto gastrointestinal y afectaría negativamente el rendimiento de carcasa (Cámara et al., 2014). Conejos alimentados con dietas con alto contenido de fibra mostraron menores rendimien- 
tos de carcasa debido a un mayor desarrollo del tracto digestivo (García et al., 2006).

El incremento de aminoácidos en un 10 y $20 \%$ con relación a lo recomendado por el $\mathrm{NRC}$ en las dietas de $2.9 \mathrm{Mcal} \mathrm{ED} / \mathrm{kg}$ no aumentó el rendimiento de carcasa, posiblemente debido a que no se estarían utilizando en los procesos de síntesis de tejido cárnico por una deficiencia en la ingestión de energía. Esto si se considera que el 3.10 y $3.40 \mathrm{~g}$ lisina/Mcal están por encima de lo recomendado por el NRC (1995), y que este aminoácido es utilizado casi exclusivamente para el crecimiento (McDonald, 1995). Los reportes de rendimiento de carcasa evaluando diferentes niveles de lisina y AA con dietas isoenergéticas $(2.75 \mathrm{Mcal} \mathrm{ED} / \mathrm{kg}$ ) bajo una alimentación de solo alimento balanceado varían entre 65.07 y $69.94 \%$ (Remigio, 2006), resultados similares a los obtenidos en la presente investigación.

No se encontró diferencias estadísticas para el peso de del hígado, riñón y corazón, pero pudo observarse que los valores más bajos correspondieron al tratamiento con 2.7 Mcal ED/kg y $100 \%$ DA con respecto al NRC (1995), cuyo aporte de nutrientes está por debajo de lo recomendado. Según Typpo et al. (1985), los cambios en el peso de los órganos sería el mejor criterio para las determinaciones del efecto del uso de niveles de aminoácidos.

\section{Conclusiones}

- El efecto de los niveles de energía digestible y la densidad de aminoácidos, en relación a las normas del NRC (1995), solo mejoró la conversión alimenticia; siendo los más eficientes el tratamiento con $2.9 \mathrm{Mcal} / \mathrm{kg}$ y $120 \% \mathrm{DA}$ ) y el tratamiento control (NRC, 1995).

- Considerando el factor energía digestible, se encontró un efecto favorable sobre la ganancia de peso, consumo de alimento, conversión alimenticia y rendimiento de carcasa con dietas de 2.9 Mcal de ED/kg de alimento.

- El incremento en 10 y $20 \%$ la densidad de aminoácidos solo mostró tendencias de mejora en los parámetros productivos evaluados.

\section{Literatura Citada}

1. Albino LFT, Silva SHM, Vargas JGV, Rostagno HS, Silva MA. 1999. Níveis de metionina mais cistina para frangos de corte de 1 a 21 e 22 a 42 dias de idade. Rev Bras Zootec 28: 519-525.

2. Alvarez $J$ L, Marguenda I, GarcíaRebollar P, Carabaño R, De Blas C, Corujo A, García-Ruiz AI. 2007. Effects of type and level of fibre on digestive physiology and performance in reproducing and growing rabbits. World Rabbit Sci 15: 9-17.

3. [AOAC] Association of Official Analytical Chemists. 2005. Official methods of analysis of the Association of Official Analytical Chemists. Ch. 34. 18th ed. Washington DC, UDSA: AOAC. 73 p.

4. Beaulieu AD, Williams NH, Patience JF. 2009. Response to dietary digestible energy content in growing pigs fed cereal grain-based diets. J Anim Sci 87: 965976. doi: 10.2527/jas.2007-0834

5. Cámara L, Berrocoso JD, Sánchez JL, López-Bote CJ, Mateos GG 2014. Influence of net energy content of the diets on productive performance and carcass merit of gilts, boars and immunocastrated males slaughtered at 120 kg BW. Meat Sci 98: 773-780. doi: 10.1016/j.meatsci.2014.07.025

6. Cerna A. 1997. Evaluación de niveles de residuos de cervecería seco en el crecimiento - engorde en cuyes. Tesis de Ingeniero Zootecnista. Lima, Perú: Univ Nacional Agraria La Molina. 85 p. 
7. Correa R. 1988. La crianza del cuy. Pasto, Colombia: Instituto Colombiano Agropecuario. $47 \mathrm{p}$.

8. Field A. 2009. Descobrindo a estatística usando SPSS. Porto Alegre, Brasil: Ed Artmed. $688 \mathrm{p}$.

9. Garcia J, Carabaño R, De Blas C, Garcia A. 2006. Importancia del tipo de fibra: nuevos conceptos y ejemplos para su aplicación en cunicultura. XXII Curso de especialización FEDNA. Barcelona. [Internet]. Disponible en: http:// www.fundacionfedna.org/publicaciones_2006

10. Kallabis KE, Kaufmann O. 2012. Effect of a high-fibre diet on the feeding behaviour of fattening pigs. Archiv Tierzucht 55: 272-284.

11. Leclercq B. 1998. Lysine: specific effects of lysine on broiler production: comparison with threonine and valine. Poultry Sci 77: 118-123. doi: 10.1093/ps/ 77.1.118

12. Mateos GG, Jiménez-Moreno E, Serrano MP, Lázaro RP. 2012. Poultry response to high levels of dietary fiber sources varying in physical and chemical characteristics. J Appl Poultry Res 21: 156-174. doi: 10.3382/japr.2011-00477

13. McDonald P. 1995. Nutrición animal. Zaragoza, España: Ed Acribia. 576 p.

14. Morales A, Carcelén F, Ara M, Arbaiza T, Chauca L. 2011. Evaluación de dos niveles de energía en el comportamiento productivo de cuyes (Cavia porcellus) de la raza Perú. Rev Inv Vet Perú 22: 177-182. doi: http://dx.doi.org/ 10.15381/rivep.v22i3.254

15. [NRC] National Research Council. 1978. Nutrient requirements of the guinea pig. In: Nutrient requirements of laboratory animals. $3^{\text {rd }}$ Revised Ed. Washington DC, USA: National Academy Press. p 59-59.
16. [NRC] National Research Council. 1995. Nutrient requirements of the guinea pig. In: Nutrient requirements of laboratory animals. $4^{\text {th }}$ Revised Ed. Washington DC, USA: National Academy Press. p 103-124.

17. Pesti GM, Bakali RI, Cervantes HM, Bafundo KW. 1999. Studies on senduramicin and nutritional responses: 2. Methionine levels. Poultry Sci 78: 1170-1176.

18. Remigio R. 2006. Evaluación de tres niveles de lisina y aminoácidos azufrados en dietas de crecimiento para cuyes (Cavia porcellus L) mejorados. Tesis de Magíster. Lima, Perú: Univ Nacional Agraria La Molina. $97 \mathrm{p}$.

19. Schutte JB, Pack M. 1995. Sulphur amino acid requirement of broiler chicken from fourteen to thirty-eight days of age. 1. Performance and carcass yield. Poultry Sci 74: 480-487. doi: 10.3382/ ps. 0740480

20. Tesseraud S, Peresson R, Lopes J, Chagneau AM. 1996. Dietary lysine deficiency greatly affects muscle and liver protein turnover in growing chickens. Br J Nutr 75: 853-865.

21. Torres R. 2006. Evaluación de dos niveles de energía y proteína en el concentrado de crecimiento de cuyes machos. Tesis de In. Zootecnista. Lima, Perú: Univ Nacional Agraria La Molina. $67 \mathrm{p}$.

22. Troesch B, Biesalski HK, Bos R, Buskens E, Calder PC, Saris WHM, Spieldenner J, et al. 2015. Increased intake of foods with high nutrient density can help to break the intergenerational cycle of malnutrition and obesity. Nutrients 7: 6016-6037. doi: 10.3390/ nu7075266

23. Typpo J, Anderson H, Krause F, Yu D. 1985. The lysine requirement of young growing male guinea pigs. J Nutr 115: 579-587. 